\title{
BMJ Open Knowledge and attitude of healthcare professionals to frailty screening in primary care: a systematic review protocol
}

\author{
ljeoma Okpechi (D), Gurch Randhawa (D), David Hewson (D)
}

To cite: Okpechi I, Randhawa G, Hewson D. Knowledge and attitude of healthcare professionals to frailty screening in primary care: a systematic review protocol. BMJ Open 2020;10:e037523. doi:10.1136/ bmjopen-2020-037523

- Prepublication history and additional material for this paper are available online. To view these files, please visit the journal online (http://dx.doi. org/10.1136/bmjopen-2020037523).

Received 05 February 2020 Revised 23 April 2020 Accepted 29 May 2020

\section{Check for updates}

(c) Author(s) (or their employer(s)) 2020. Re-use permitted under CC BY-NC. No commercial re-use. See rights and permissions. Published by BMJ.

Institute for Health Research, University of Bedfordshire, Luton, UK

Correspondence to Dr Gurch Randhawa; gurch.randhawa@beds.ac.uk

\section{ABSTRACT}

Introduction Frailty is an increasingly common condition in which physiological decline as a result of accumulated deficits renders older people more vulnerable to adverse outcomes. An increasing range of frailty screening programmes have been introduced in primary care to identify frail older people in order to deliver appropriate interventions. However, limited information on the knowledge and attitude of healthcare professionals (HCPs) with respect to frailty screening is known. The aim of this systematic review is to provide evidence on the knowledge and attitude of HCP in terms of frailty screening, and potentially identify barriers and facilitators to frailty screening to improve implementation of frailty screening in primary care.

Methods/design A systematic review of qualitative research will be conducted. Databases searched will be MEDLINE, Cumulative Index to Nursing and Allied Health Literature, PsycINFO and Web of Science from January 2001 to August 2019. Methods will be reported based on the Preferred Reporting Items for Systematic Reviews and Meta-Analyses. Population, interest, context and study design methodology was used to develop inclusion and exclusion criteria with HCPs as population, frailty screening as interest and knowledge or attitude of HCPs to frailty screening as context. Studies with a qualitative methodology or a mixed-method design where the qualitative component is analysed separately will also be included. Quality appraisal will be carried out using the Joanna Briggs Institute appraisal tool for qualitative studies. Data will be extracted from each selected study with thematic framework analysis used to synthesise findings.

Ethics and dissemination This systematic review does not require ethical approval as primary data will not be collected. The findings will be disseminated at conferences and in a relevant academic journal. This review will assist HCPs and relevant stakeholders to tackle the challenges of frailty screening in primary care.

PROSPERO registration number CRD42019159007.

\section{INTRODUCTION}

The increase in the ageing population means there is an urgent need to focus on conditions that affect older people, such as frailty. ${ }^{1}$ Frailty is a condition that is prevalent in older
Strengths and limitations of this study

- This systematic review protocol will decrease the likelihood the research will be duplicated unnecessarily and improve quality due to peer review.

- This will be the first systematic review to study the knowledge and attitude of healthcare professionals (HCPs) to frailty screening in primary care.

- The review will not consider HCPs' knowledge and attitude in studies conducted in secondary or tertiary care settings.

people, whereby the gradual decrease in multiple physiological functions means an individual's system can no longer maintain homeostasis. ${ }^{2}$ The consequence of frailty is an increased susceptibility to adverse health outcomes. ${ }^{3}$ The complexity associated with frailty has made the identification and management of this condition challenging for healthcare professionals (HCPs). ${ }^{4}$

There have been recommendations to implement frailty screening in primary care as a policy. For instance, the Canadian Frailty Network recommends that screening is a part of routine practice, ${ }^{5}$ while there has also been a push within Australia for a policy directive on frailty screening to be introduced. ${ }^{6}$ The UK was the first country to implement a national screening programme for frailty. ${ }^{7}$ This policy was included as part of the General Medical Services contract in 2017, in which the National Health Service requires general practitioners (GPs) to screen all people aged 65 years and over, using the electronic Frailty Index (eFI). ${ }^{8}$ The eFI uses electronic health record data to construct a Frailty Index based on the accumulation of deficits model. ${ }^{9}$ Patients are categorised as fit, mildly frail, moderately frail or severely frail, based on the number of deficits. ${ }^{8}$ Other frailty screening tools such as the Preferred Reporting Items for Systematic Reviews and 
Meta-Analyses (PRISMA)-7 have been recommended for use in primary care. ${ }^{10} \mathrm{~A}$ key part of any screening process, regardless of the tool used, is the use of clinical judgement in the screening process. ${ }^{11}$

The choice of GP practices to screen for frailty has been suggested as a viable solution due to the easy accessibility to patients and the trustworthy relationship established in such primary care settings. ${ }^{12}$ In addition to GPs, the use of a multidisciplinary team (MDT) approach to tackle the complexity of managing frail patients has also been proposed. ${ }^{13}$ The MDT in this setting could include a range of HCPs such as pharmacists, practice nurses, community nurses, geriatric nurses, social workers, occupational therapists and physiotherapists. ${ }^{14} 15$

However, as with other screening programmes, implementation can cause reluctance. Reeves $e t a l^{7}$ suggested that some GPs perceived frailty screening to be difficult and another burden on what is already a demanding job. As an example, a typical GP practice in the UK with 2000 older patients, based on the original eFI data, ${ }^{8}$ would have $320(16 \%)$ moderately frail patients and $80(4 \%)$ severely frail patients. Other challenges to the implementation of frailty screening include the lack of an operational definition of frailty, the complexity of frailty as a concept, the choice of an appropriate screening tool to adopt, the effectiveness of the tool and how easy it is to use. ${ }^{1216}$ Further concerns have been raised about the time taken for screening and the process of care management, not to mention the increased cost and burden on GPs. ${ }^{17}$

There is also evidence that positive attitudes from nursing participants when using the Frailty Assessment for Care planning Tool produced an effective result. ${ }^{18} \mathrm{It}$ is important, therefore, to consider the attitude and knowledge of HCP performing this screening to determine the acceptability and efficiency of implementing a screening programme to identify frail adults. ${ }^{16}$

There have been several recent systematic reviews in which frailty screening in primary care has been assessed. ${ }^{19}{ }^{20}$ However, none of these reviews focused on the views of the HCP who are carrying out frailty screening, instead assessing only the effectiveness of the screening programme. Therefore, the need to explore the knowledge and attitude of HCPs towards frailty screening is essential.

The aim of this systematic review is to determine the knowledge and attitude of HCPs towards frailty screening in primary care. Findings from this review will not only inform policy but could also provide wider perspectives that will be analysed to improve frailty screening in primary care. In addition to knowledge and attitudes, identifying the barriers and facilitators affecting the adoption of frailty screening in primary care could also improve implementation of frailty screening.

\section{METHODS AND DESIGNS}

The search strategy was developed according to the PRISMA Protocols. ${ }^{21}$ In addition, due to the qualitative approach taken in this review, the enhancing transparency in reporting the synthesis of qualitative research statement will be adhered to. ${ }^{22}$

\section{Selection criteria}

This review will include studies that have addressed the views of healthcare practitioners with respect to frailty screening in primary care. The type of studies that will be included are qualitative designs including action research, grounded theory, ethnography and phenomenology. Mixed methods studies will be accepted only if the qualitative findings are reported separately from quantitative results. Data collection using both focus groups and interviews will be accepted.

Inclusion criteria for the review were developed based on the population, interest, context and study design (PICoS) methodology for qualitative reviews. ${ }^{23}$ With respect to the population, participants in must be HCP including practice nurses, doctors and physiotherapists, providing the HCP are working in a primary care setting. The National Institute for Health and Care Excellence definition of primary care as care services delivered outside the walls of the hospitals with a range of service providers will be used. ${ }^{24}$ This will include health centres, walk-in centres and community clinics. Studies focusing on non-HCPs such as stakeholders, policy makers, health assistants and student nurses will be excluded from the review. The phenomenon of interest is the concept of frailty screening, regardless of the screening tools used by HCPs working in primary care, while the context is studies that describe and explore HCPs' views on frailty screening, including barriers and facilitators to adoption. A summary of the PICoS inclusion and exclusion criteria is shown in table 1 .

\section{Search strategy}

The literature search will use a three-step process, as recommended by the Joanna Briggs Institute (JBI) ${ }^{25}$ The first stage of this process requires keywords to be identified by the reviewers, which are then augmented by words from the titles and abstracts of relevant primary studies. A comprehensive search strategy will be conducted using Boolean operators, Medical Subject Heading (MeSH) terms and date limiters as search filters to identify relevant qualitative studies. ${ }^{26} \mathrm{~A}$ list of the keywords such as frailty, screening, attitude and HCPs specific to each database with related words and terms similar to the outlined keywords was developed. These will include views, perception, experience and belief, including variations of search terms adopted in similar systematic reviews (online supplementary appendix 1). The second stage consists of the creation of database-specific keyword searches. The databases that will be searched will be MEDLINE, PsycINFO, Cumulative Index to Nursing and Allied Health Literature and the Web of Science Core Collection. The third stage will be hand searching reference lists of the selected articles retrieved in phase two. In addition, selected specialist articles in the field on frailty research 


\begin{tabular}{lll}
\hline \multicolumn{2}{l}{ Table 1 Inclusion and exclusion criteria } & \\
\hline PICoS strategy & Inclusion criteria & Exclusion criteria \\
\hline P-Population & HCP working in primary care settings & $\begin{array}{l}\text { Primary care workers who are not HCPs } \\
\text { HCPs working in any other care setting }\end{array}$ \\
I-phenomenon of Interest & Frailty screening & \\
Co-Context & $\begin{array}{l}\text { Knowledge and attitude of HCPs on frailty } \\
\text { screening }\end{array}$ & \\
S-Study design & All qualitative study designs & Non-primary literature and other study designs \\
\hline
\end{tabular}

HCPs, healthcare professionals.

will be hand searched for relevant studies. Publication date will be limited to dates between the year 2001, when the frailty phenotype was first published, ${ }^{27}$ and August 2019, while only studies in English will be included.

\section{Study selection}

Articles identified by the keyword search will be combined, with duplicates removed using EndNote bibliographic software (V.9, Clarivate Analytics, Philadelphia, Pennsylvania, USA). Two reviewers (IO and DH) will screen titles and abstracts independently to identify relevant studies using the eligibility criteria specified above, with articles marked as yes/no/maybe. Any articles with yes/ yes or yes/maybe will be advanced to full text review. Any conflicts of yes/no and no/maybe will be resolved by the third author (GR).

\section{Data extraction and management}

Data will be extracted from full-text versions of the articles using a Microsoft Excel spreadsheet based on the JBI Qualitative Assessment and Review Instrument (QARI) data extraction form for interpretive and critical research. ${ }^{25}$ Data extracted using this method will include first author, year and title, the population, geographical location, study methods and the phenomena of interest relevant to the review question and specific objectives. These will include the frailty screening tool or assessment used, and the views on knowledge and attitude identified by the HCPs that are deemed relevant to the research question. All data will be extracted by both $\mathrm{IO}$ and $\mathrm{DH}$ independently, then agreed and combined. Disagreements will be resolved by GR.

\section{Quality appraisal}

All studies will be quality appraised by $\mathrm{IO}$ and $\mathrm{DH}$ independently using the JBI critical appraisal checklist for qualitative research. ${ }^{25}$ This tool consists of 10 questions, each of which is scored as 0 or 1 . There are questions that address validity, ethical considerations, transparency and congruity between study philosophical stance. Each of the questions is given a yes or no answer, with studies then rated as 1 or 0 for yes and no, respectively. The sum of points after grading will be used to determine quality, with studies scoring 0-3 considered to be of poor quality, studies scoring 4-6 considered to be moderate, while and studies scoring
7-10 will be considered to be of high quality. Disagreements in initial ratings of methodological quality will be resolved by a third reviewer (GR) and discussed until an agreement is reached.

\section{Data synthesis and analysis plan}

To synthesise and analyse the results in this review, a thematic analysis approach will be used. ${ }^{28-30}$ This form of approach allows large descriptive evidence to be explored and establish an understanding of the knowledge and attitude of HCPs towards frailty screening in primary care. The different stages of framework synthesis will be followed to synthesise the qualitative data, as detailed below:

Familiarisation with the data: The first author will become acquainted with data to meet the aims of the review and identify emerging themes across the studies.

Identifying a thematic framework: The thematic analysis approach outlined by the JBI for qualitative synthesis will be adopted to capture and guide emerging themes, without using coding software. ${ }^{25}$ The manual provides detailed information on identification of relevant themes.

Indexing: The first author will independently read the selected articles and extractions to identify themes. The framework will be reviewed to capture common and significant phenomenon as new themes emerge. There will be room for discussion and agreement among research team members. Data will be grouped, and coding will be done on themes identified using a Microsoft Excel sheet. Each study will be indexed using codes related to the themes.

Data display: Data and themes or patterns identified will be assembled, organised and concise to fit into a table or itemised with textual display to draw conclusion easily.

Interpretation: Themes identified will be mapped to capture the phenomena that needs exploration to determine the association between themes and aid explain the findings clearly. Findings will be structured to meet the aim and objective of the review with the emerging themes.

Summary and findings verification: This will be presented to draw reasonable conclusions from key findings using a reflective process. This will then be used to confirm whether the findings have been able to answer the research questions and draw implications from it. 


\section{Patient and public involvement}

This protocol was drafted without public or patient involvement. However, relevant stakeholders will be included in the interpretation of the findings from this review, as well as in the development of strategies for dissemination. will be made available to HCPs, policymakers, patients and the public.

\section{DISCUSSION}

Frailty is an increasingly prevalent condition that clinicians will need to deal with, particularly as the older population continues to increase. ${ }^{16}$ One of the most effective ways of reducing the prevalence of frailty is to screen older people early enough to prevent the condition from causing adverse outcomes. ${ }^{1}$ Unfortunately, there are gaps that distort the flow of screening as part of routine practice in primary care. ${ }^{17}$ The level at which screening for frailty is embraced by physicians and other healthcare practitioners is important for adequate service delivery. ${ }^{12}$

The studies included will have approached this topic using qualitative methods to explore the phenomenon of interest. This review will explore the attitude and knowledge of the different clinicians and HCP towards frailty screening. This will add strength to the process of developing more efficient models for general practice including ways to overcome barriers to screening in primary care. Views from a multidimensional approach might also be more effective than those from a single approach or from one HCP specialty. Also, considering how specific screening tools can facilitate screening implementation in primary care will add value to this review. However, including studies conducted within the primary care setting and excluding the hospital area could serve as a limitation to this review, with some screening for frailty taking place in this setting. Also, not including other participants such as older adults and their carers is another limitation to this review. Furthermore, the reviewer intends to analyse data for interpretation thematically for substantial and quality evidence, which could also introduce bias.

This review will be the first of its kind and therefore seeks to add to the body of knowledge around the attitude and knowledge of HCPs to frailty screening in primary care. The findings from this review will potentially highlight barriers to frailty screening and possibly identify facilitators to improve the implementation of routine screening for frailty in primary care. These barriers and facilitators will be disseminated widely and will also be presented to stakeholders within the UK to enable screening practices to be improved. Finally, and most importantly, findings will assist healthcare practitioners to provide optimum care service to older patients.

\section{ETHICS AND DISSEMINATION}

Ethical approval will not be needed as this review will obtain information from other studies. The aim is to disseminate the findings of this review through publication in a peer-reviewed journal and presentation at relevant conferences. The findings from this review will also be disseminated to relevant stakeholders via a report and summary in lay language, and presentation at key networks, which will boost the impact of this review.

\section{Study status}

The review is ongoing and is expected to be completed by June 2020 .

Contributors 10 performed the initial search that was presented to $\mathrm{DH}$ as a systematic review specialist, who refined the search strategy to cover a wider scope. DH and GR reviewed the first draft and made suggestions. The final manuscript was drafted and approved by all authors.

Funding The authors have not declared a specific grant for this research from any funding agency in the public, commercial or not-for-profit sectors.

Competing interests None declared.

Patient and public involvement Patients and/or the public were not involved in the design, or conduct, or reporting, or dissemination plans of this research.

Patient consent for publication Not required.

Provenance and peer review Not commissioned; externally peer reviewed.

Open access This is an open access article distributed in accordance with the Creative Commons Attribution Non Commercial (CC BY-NC 4.0) license, which permits others to distribute, remix, adapt, build upon this work non-commercially, and license their derivative works on different terms, provided the original work is properly cited, appropriate credit is given, any changes made indicated, and the use is non-commercial. See: http://creativecommons.org/licenses/by-nc/4.0/.

\section{ORCID iDs}

ljeoma Okpechi http://orcid.org/0000-0002-0850-1112

Gurch Randhawa http://orcid.org/0000-0002-2289-5859

David Hewson http://orcid.org/0000-0002-7656-4000

\section{REFERENCES}

1 Clegg A, Young J, Iliffe S, et al. Frailty in elderly people. Lancet 2013;381:752-62.

2 Dent E, Kowal P, Hoogendijk EO. Frailty measurement in research and clinical practice: a review. Eur J Intern Med 2016;31:3-10.

3 Bergman H, Ferrucci L, Guralnik J, et al. Frailty: an emerging research and clinical paradigm--issues and controversies. J Gerontol A Biol Sci Med Sci 2007;62:731-7.

4 Fairhall N, Langron C, Sherrington C, et al. Treating frailty--a practical guide. BMC Med 2011;9:83.

5 Grimes K, Kitts J, Tholl B, et al. Policy and economic considerations for frailty screening in the Canadian healthcare system. J Frailty Aging 2018;7:233-9.

6 Ambagtsheer RC, Beilby JJ, Visvanathan R, et al. Should we screen for frailty in primary care settings? A fresh perspective on the frailty evidence base: a narrative review. Prev Med 2019;119:63-9.

7 Reeves D, Pye S, Ashcroft DM, et al. The challenge of ageing populations and patient frailty: can primary care adapt? BMJ 2018;362:k3349.

8 Clegg A, Bates C, Young J, et al. Development and validation of an electronic frailty index using routine primary care electronic health record data. Age Ageing 2016;45:353-60.

9 Rockwood K, Mitnitski A. Frailty in relation to the accumulation of deficits. J Gerontol A Biol Sci Med Sci 2007;62:722-7.

10 Abbasi M, Rolfson D, Khera AS, et al. Identification and management of frailty in the primary care setting. CMAJ 2018;190:E1134-40.

11 Hoogendijk EO, van der Horst $\mathrm{HE}$, van de Ven PM, et al. Effectiveness of a geriatric care model for frail older adults in primary care: results from a stepped wedge cluster randomized trial. Eur J Intern Med 2016;28:43-51.

12 Drubbel I, de Wit NJ, Bleijenberg N, et al. Prediction of adverse health outcomes in older people using a frailty index based on routine primary care data. J Gerontol A Biol Sci Med Sci 2013;68:301-8. 
13 Metzelthin SF, Daniëls R, van Rossum E, et al. A nurse-led interdisciplinary primary care approach to prevent disability among community-dwelling frail older people: a large-scale process evaluation. Int J Nurs Stud 2013;50:1184-96.

14 Gustafsson S, Edberg A-K, Dahlin-Ivanoff S. Swedish Health Care Professionals' View of Frailty in Older Persons. J Appl Gerontol 2012;31:622-40.

15 Riley R, Coghill N, Montgomery A, et al. Experiences of patients and healthcare professionals of NHS cardiovascular health checks: a qualitative study. J Public Health 2016;38:543-51.

16 Rodríguez-Laso Ángel, O'Caoimh R, Galluzzo L, et al. Population screening, monitoring and surveillance for frailty: three systematic reviews and a grey literature review. Ann Ist Super Sanita 2018;54:253-62.

17 Shaw RL, Gwyther H, Holland C, et al. Understanding frailty: meanings and beliefs about screening and prevention across key stakeholder groups in Europe. Ageing Soc 2018;38:1223-52.

18 Moffatt $\mathrm{H}$, Moorhouse $\mathrm{P}$, Mallery L, et al. Using the frailty assessment for care planning tool (fact) to screen elderly chronic kidney disease patients for frailty: the nurse experience. Clin Interv Aging 2018;13:843-52.

19 Lee L, Patel T, Hillier LM, et al. Identifying frailty in primary care: a systematic review. Geriatr Gerontol Int 2017;17:1358-77.

20 Pialoux T, Goyard J, Lesourd B. Screening tools for frailty in primary health care: a systematic review. Geriatr Gerontol Int 2012;12:189-97.
21 Moher D, Shamseer L, Clarke M, et al. Preferred reporting items for systematic review and meta-analysis protocols (PRISMA-P) 2015 statement. Syst Rev 2015;4:1.

22 Tong A, Flemming $\mathrm{K}$, Mclnnes $\mathrm{E}$, et al. Enhancing transparency in reporting the synthesis of qualitative research: ENTREQ. BMC Med Res Methodol 2012;12:181.

23 Lockwood C, Munn Z, Porritt K. Qualitative research synthesis. Int J Evid Based Healthc 2015;13:179-87.

24 NICE. Primary care. National Institute for Health and Care Excellence, 2019.

25 Aromataris E, Munn Z. Joanna Briggs Institute reviewer's manual. The Joanna Briggs Institute, 2017. https://reviewersmanual. joannabriggs.org/

26 Aveyard H. Doing a literature review in health and social care: a practical guide. McGraw-Hill Education (UK), 2014.

27 Fried LP, Tangen CM, Walston J, et al. Frailty in older adults: evidence for a phenotype. J Gerontol A Biol Sci Med Sci 2001;56:M146-57.

28 Ochodo E, Kredo T, Young T, et al. Protocol for a qualitative synthesis of barriers and facilitators in implementing guidelines for diagnosis of tuberculosis. BMJ Open 2017;7:e013717.

29 Thomas J, Harden A. Methods for the thematic synthesis of qualitative research in systematic reviews. BMC Med Res Methodol 2008;8:45.

30 Braun V, Clarke V. Using thematic analysis in psychology. Qual Res Psychol 2006;3:77-101. 